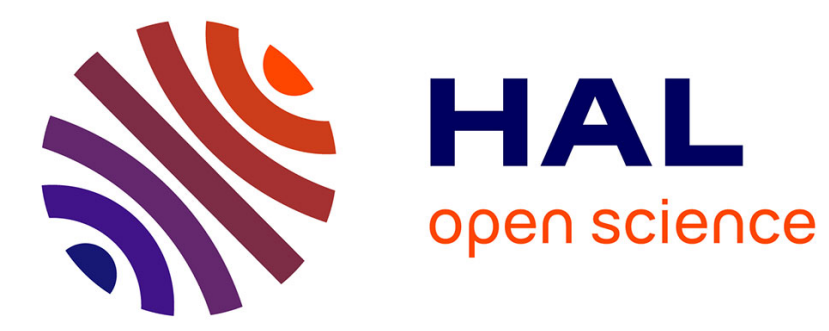

\title{
Position isomerism on one and two photon absorption in multibranched chromophores: a TDDFT investigation.
}

\author{
Claudine Katan, Mireille Blanchard-Desce, Sergei Tretiak
}

\section{To cite this version:}

Claudine Katan, Mireille Blanchard-Desce, Sergei Tretiak. Position isomerism on one and two photon absorption in multibranched chromophores: a TDDFT investigation.. Journal of Chemical Theory and Computation, 2010, 6 (11), pp.3410-3426. 10.1021/ct1004406 . hal-00559420

\section{HAL Id: hal-00559420 \\ https://hal.science/hal-00559420}

Submitted on 25 Nov 2016

HAL is a multi-disciplinary open access archive for the deposit and dissemination of scientific research documents, whether they are published or not. The documents may come from teaching and research institutions in France or abroad, or from public or private research centers.
L'archive ouverte pluridisciplinaire HAL, est destinée au dépôt et à la diffusion de documents scientifiques de niveau recherche, publiés ou non, émanant des établissements d'enseignement et de recherche français ou étrangers, des laboratoires publics ou privés. 


\title{
Position isomerism on one and two photon absorption in multibranched chromophores: a TDDFT investigation.
}

\author{
Claudine Katan*a,b, Mireille Blanchard-Desce ${ }^{\mathrm{a}}$ \\ ${ }^{a}$ Université Européenne de Bretagne, \\ CNRS - Chimie et Photonique Moléculaires (CPM), \\ Université de Rennes 1, 35042 Rennes, France \\ ${ }^{b}$ Université Européenne de Bretagne, \\ CNRS - Fonctions Optiques pour les Technologies de l'informatiON (FOTON), \\ INSA de Rennes, CS70839, 35708 Rennes, France \\ Sergei Tretiak ${ }^{\mathrm{c}}$ \\ ${ }^{c}$ Center for NonLinear Studies (CNLS), and \\ Center for Integrated NanoTechnologies (CINT), \\ Los Alamos, NM USA 87545
}

\begin{abstract}
:
Recently, branching and click chemistry strategies have been combined to design a series of optically active chromophores built from triazole moieties. These triazole based multipolar chromophores have shown to be promising candidates for two-photon absorption (TPA) transparency optimization in perspective of optical limiting in the visible region. In this work, the nature of one- and two-photon absorption properties in a family of triazole based chromophores has been investigated using hybrid time dependent density functional theory (TD-DFT). We use recent extensions of TD-DFT to determine nonlinear optical responses, and Natural Transition Orbitals to analyze the underlying electronic processes. Our results are also interpreted in the framework of the Frenkel exciton model. In agreement with experimental data, we found that introducing triazole moiety into multibranched chromophores substantially modifies their optical behavior due to changes in electronic delocalization and charge-transfer properties between donating end-groups and branching center that can be controlled by the triazole ring. Structural conformations via modulation of the torsion between phenyl and triazole rings significantly alter excited state electronic structure. Moreover, isomer positioning also greatly influences both linear and nonlinear optical responses such as TPA. Our theoretical findings allow elucidating these differences and contribute to the general understanding of structure-property relations. Consequently, interplay of donor/acceptor strength, triazole regioisomerism, and branching are shown to provide flexible means allowing for precise tuning of both linear and nonlinear optical responses, thus opening new perspectives towards synergic TPA architectures.
\end{abstract}

Keywords: two-photon absorption, TPA, NLO, time dependent density functional theory, TD-DFT, Frenkel exciton model, triazole, multibranched chromophores 


\section{I.Introduction}

1,2,3-triazole chemistry has recently gained renewed interest thanks to the click methodology offering far more ease of synthesis. ${ }^{1,2}$ This has prompt synthetic effort to design novel molecular architectures in which the triazole moiety is assumed to play quite different roles, such as electron donor, ${ }^{3}$ electron acceptor, ${ }^{2,4,5}$ active $\pi$-linker, ${ }^{6,7}$ metal ligating entity ${ }^{8,9}$ or siteisolation group. ${ }^{10-12}$ In particular, 1,2,3-triazole click chemistry has been explored in the field of small push-pull ${ }^{3,6,9,13}$ and larger multipolar ${ }^{4,13}$ chromophores up to dendrimers ${ }^{10}$ and polymers with linear ${ }^{5}$ or hyperbranched ${ }^{7,11,12}$ polytriazole structures. In addition, 1,2,3-triazole has four points of attachment that allows for the design of various regiosiomers which may show quite different behaviors.

This recent synthetic effort was partly driven by interest for their photophysical properties which have long been overlooked and are still underdeveloped. ${ }^{6}$ Recently, based on the assumption of electron-donating properties of triazole, ligation of this ring has been shown to effectively modulate the emission properties of coumarin leading to increased fluorescence quantum yield by more than an order of magnitude. ${ }^{3}$ Investigation of the photophysical properties of isomeric donor-acceptor-substituted 1,2,3-triazoles has also been reported. ${ }^{6}$ It has been demonstrated both experimentally and theoretically that positioning of the donor and acceptor on the triazole ring results in important modulations of both transition energies and absorption intensities. ${ }^{6}$ In the case of 1,4 and 1,5-linked hyperbranched polytriazoles, the different emission colors of the regiostructures were related to the differences in aggregation in the solid state. ${ }^{7}$ Based on the site-isolation concept, ${ }^{14}$ different hyperbranched polytriazoles have shown enhancement of their second order non linear optical (NLO) responses overcoming the asymptotic dependence of electro-optic activity on chromophore density. ${ }^{10-}$ ${ }^{12,15}$ Finally, triazole based multipolar ${ }^{4}$ and functional poly(aroyltriazole) $\mathrm{s}^{5}$ have also been promises for their nonlinear optical properties, in particular, two photon absorption (TPA) capacity.

Indeed, among the numerous NLO processes, simultaneous absorption of two photons has gained increased attention over recent years ${ }^{16-21}$ as it opens the way for improved and novel technological capabilities. This, in turn, led to the search for adequate functional materials with enhanced TPA responses. Thanks to their inherent modularity and potentially high TPA responses, conjugated organic molecular systems are of particular interest. In that perspective, the branching strategy has received much attention over recent years. ${ }^{16-18}$ It relies on multibranch systems obtained by gathering several molecular units which are either of $\operatorname{dipolar}^{21-31}$ (also known as push-pull chromophores) or quadrupolar ${ }^{32-38}$ type. In such nanoarchitectures, the interbranch coupling plays a central role. Depending on the nature and the strength of the interaction between the molecular units, optical properties such as TPA can show cooperative enhancement, additive behavior or weakening. The interchromophore interactions are related to quite a few structural parameters: nature and length of the monomeric building blocks, number of chromophoric units as well as nature of the branching center. All these parameters will directly influence inter-monomer distances and orientation and control the overall molecular symmetry. ${ }^{27}$ Symmetry will, in turn, result in optically allowed and forbidden electronic transitions and drive oscillator strength redistribution among excited states (ESs) when going from the monomeric unit to the multichromophoric architecture. Besides the nature of the excitation, these parameters also influence the subsequent excitation energy transfer dynamics. ${ }^{39}$ 
Recently, branching and click chemistry strategies have been combined to design a series of TPA active chromophores built from triazole moieties. ${ }^{4,13,40,41}$ These triazole based multipolar chromophores have shown to be promising candidates for TPA transparency optimization in perspective of optical limiting in the visible region. Obtained quadrupolar and three-branched chromophores have shown to combine efficient synthesis, high solubility, intense absorption in the near UV region, full transparency, high excited state lifetimes and good TPA optical responses. ${ }^{4,13}$ Moreover, emission wavelengths can be tuned and excited state lifetimes can be significantly increased by increasing the polarity of the solvent. Related derivatives such as four branched systems or position isomers have also been designed and experimentally investigated. ${ }^{40,41}$ Experimental data reveal quite different behaviors as compared to the related quadrupolar, octupolar or other multibranched compounds for both linear and NLO responses. As an example, when compared to similar three branched systems also built by gathering branches through a triphenylamine central core, the triazole derivative deviates significantly. Indeed, no marked solvatochromic behavior of emission has been observed while it is very effective for the quadrupolar analog ${ }^{13,40}$ for which symmetry breaking in the relaxed excited state is thus clearly evidenced. ${ }^{42}$ In addition, TPA cross sections of the three branched chromophore still increases at $577 \mathrm{~nm}$. ${ }^{4}$ Thus, no maximum is observed near twice the one photon absorption (OPA) wavelength $(694 \mathrm{~nm})$ as is usually the case.

To get a better understanding of triazole based chromophores and to provide a deep physical insight into ongoing phenomena, theoretical calculations can be quite useful. Among the many different approaches ranging from simple few states models up to high level quantum methods (QM), the Frenkel exciton model has been shown to provide a valuable qualitative tool $1^{25,26,38,43-47}$ with a few exceptions. Indeed, to be applicable, not only the strength of the coupling (solely due to Coulomb interactions) between monomeric units should be weak but the inherent nature (e.g. symmetry) of the building blocks should not be affected by the branching center. ${ }^{37}$ Among the different QM methods available nowadays, adiabatic timedependent density functional theory (TD-DFT) is currently the method of choice for calculating the ES of sizeable molecular systems. ${ }^{48-55}$ Arbitrary frequency-dependent NLO polarizabilities can be subsequently derived based on the quasi-particle formalism of the TDKohn-Sham equations. ${ }^{56}$ This method has been applied to calculate OPA and TPA responses of various substituted chromophores. ${ }^{17,25,26,31,35,37,57-63}$ These studies have shown the good performances of the TD-DFT based hybrid functionals for molecular NLO properties.

In this paper we present a theoretical investigation, mainly based on the mentioned above density matrix formalism of TD-DFT for NLO responses, ${ }^{17}$ of two 1,2,3-triazole based series compiling a total of nineteen compounds (Figure 1). The case of dai, dfi, 2dai and 3dfi has already been analyzed in details elsewhere ${ }^{31}$ and we recall here the main findings as well as further interpretation for the sake of comparison to other nd and ndi chromophores. Our purpose is to address two main points: (i) the branching effect which appears to show a quite different behavior than that reported for analogous multibranched chromophores and (ii) the influence of position isomerism on both OPA and TPA responses. To our knowledge, it is the first time that TPA properties are investigated on 4-members branched chromophores ${ }^{64,65}$ as well as in relation with regioisomerism. Our findings will also be analyzed in the framework of the Frenkel exciton model. After a brief description of computational details, already well documented, we will first validate our computational approach for both series of chromophores. This is thought to allow inference to what extent comparison to experiment is reasonable, i.e. underline all sources of possible discrepancies. After analysis of the main geometrical features, we will sequentially investigate OPA and TPA responses. For both properties, we first analyze trends within each of the two series and subsequently discuss 
effects of position isomerism. Where appropriate, comparison to related branched structures will be made. The main findings will be summarized in the concluding section. We believe that this detailed theoretical study performed on a large set of compounds contributes to the overall understanding of structure-properties relationships of NLO-chromophores and opens new perspectives for further improvements within the branching strategy.

\section{II.Computational methodology}

A set of nineteen chromophores, sketched in Figure 1, have been theoretically investigated. They are classified in two different series, series nd and corresponding 1,2,3-triazole position isomers ndi. For the sake of simplicity and reduction of the numerical cost, the hexyl solubilizing chains of original chromophores ${ }^{4,13,40,41}$ have been replaced by methyl groups. These two series contain nine dipoles, three quadrupoles, five three-branched chromophores and two four-branched compounds. All QM calculations have been performed using either Gaussian $98^{66}$ or Gaussian 03 packages ${ }^{67}$ in vacuum. For ground state geometries (Figures 2 , $\mathrm{S} 1$ and S2), it was previously found that the Hartree-Fock (HF) method is superior to the DFT based approaches by reproducing bond length alternation in similar conjugated molecules. ${ }^{57} \mathrm{It}$ was also shown to be a good compromise for subsequent calculation of optical spectra. ${ }^{17,25,26,37,57-60,63}$ Thus, all ground state geometries have been optimized at the HF/6-31G level of theory, starting from a few different conformations but no exhaustive sampling have been conducted.

Linear and nonlinear optical responses have been calculated using the density matrix formalism as described in ref. 56. Subsequent computation of TPA cross sections is detailed in ref. 17. We retained the TD-B3LYP/6-31G//HF/6-31G level of theory, in conventional quantum chemical notation "single point//optimization level", for the calculation of optical spectra as it showed to be a good compromise between numerical resources and accuracy and led to quite good agreement with experimental results for a large set of related chromophores. The forthcoming section will be devoted to discuss the validity of such an approach for the present triazole-based chromophores and include comparison to results obtained with a larger amount of orbital exchange, namely the hybrid BHandH functional instead of B3LYP. The calculated ES structures includes 20, 30, 40 and 60 ESs (?) respectively for dipolar, quadrupolar, three-branched and four-branched compounds. The number of ESs (?) was checked for at least one dipole, quadrupole and octupole. It was found to be sufficient as asymptotic values are reached for the absorption spectra and only small effects (the largest deviation observed is $12 \%$ for the first band of 3dbi when using 24 instead of $40 \mathrm{ES}$ ) are discerned on the amplitude of the TPA spectra.

Besides the number of ES, a few other parameters influence the calculated response amplitudes. First, the damping factor introduced to simulate finite linewidth in the resonant spectra has been fixed to $0.20 \mathrm{eV}$ for all chromophores, on the basis of typical broadening seen in available experimental absorption spectra (Table S1). Next, no solvation effects have been accounted for in the present work. In fact, due to different solute and solvent polarizabilities, the commonly used Lorentz local field factor correction is clearly inappropriate. In addition, relevant treatment of solvent effects is more time consuming and may lead to further charge transfer overestimation. ${ }^{17}$ Last, no vibrational contributions have been considered. All these effects will influence computed OPA and TPA spectra and these approximations should always been kept in mind when comparison to experimental findings is thought. 
Natural transition orbital (NTO) analysis of the $\mathrm{ES}^{68}$ has been used to analyze the nature of the ES involved in the photophysical processes of interest. They offer a compact representation of transition densities in terms of their expansion into single-particle transitions. Figures showing molecular geometries and NTOs were obtained with XcrySDen. ${ }^{69}$ Use of Frenkel exciton model within the multibranch strategy is already well documented and the reader may refer to ref. 17 for quadrupoles and three-branched chromophores and to ref. 43 for three- and four-branched compounds.

\section{III.Results and discussion}

\section{Validation of TD-DFT approach}

First, let us validate the TD-DFT methodology used to determine the optical responses of the chromophores investigated in this work. Comparison of TD-B3LYP/6-31G//HF/6-31G results with available experimental photophysical data in toluene ${ }^{4,13}$ showed that the employed level of theory fits nicely for members of ndi series. ${ }^{31}$ Further comparison to experimental data ${ }^{40}$ for dai, $3 \mathbf{d b i}$ and 4 dei leads to comparable agreement with a calculated first absorption band slightly blue shifted with respect to the experimental band position (Table 1). Such a good agreement is not observed for members of nd series for which the calculated transition energies of the first absorption band are red shift by more than $0.3 \mathrm{eV}$ with respect experimental ones (Table 1). In addition, experimental absorption spectra of 2da and $3 \mathbf{d b}$ show two bands in the $285-400 \mathrm{~nm}$ spectral region. ${ }^{40}$ For both compounds, the first one shows up near $3.5 \mathrm{eV}$ and has a molar extinction coefficient two to three times smaller than the strongest absorption band visible near $4.2 \mathrm{eV} .{ }^{40}$ Within TD-B3LYP/6-31G//HF/6$31 \mathrm{G}$, both the ratio between oscillator strengths and the band splitting are significantly overestimated (Table 1, Figures 3, S7, S8). Similar deviations have already been reported for a series of related 1,2,3-triazole based CT regioisomers ${ }^{6}$ at TD-B3LYP/6-31G(d)//B3LYP/6$31 \mathrm{G}(\mathrm{d})$ for which agreement of experimental and calculated band position and amplitude much depends on the attachment points of substituents. Here, we may encounter the wellknown TDDFT problem related to improper description of excited state with pronounced charge transfer (CT) character. Even hybrid functionals with moderate amount of orbital exchange such as B3LYP model are affected by this problem by placing the CT states energetically too low and by miscalculating their optical transition dipoles compared to experiment. ${ }^{54}$ Functionals that contain a larger fraction of Fock-like orbital exchange have been shown to overcome this problem but at the expense of accuracy for the absolute band positions. ${ }^{54}$ Indeed, using the early hybrid BHandH functional $(50 \%$ of orbital exchange; TDBHandH/6-31G//HF/6-31G results are available as Supporting Information), leads to an overall blue shift of the first absorption band and to large deviation from experimental band position (Figures S3, S7, S8). At the same time, the splitting between the first two absorption bands becomes comparable to experimental findings while the ratio of corresponding oscillator strengths becomes close to unity, and thus becomes too small (Figure S3). As a consequence, the correct empirical amount of orbital exchanges for given compounds lies somewhere in between B3LYP and BHandH hybrid functionals. In addition, NTOs obtained within these two hybrid schemes, for a larger set of nd compounds (Figures S7-S10), show an overall good correspondence, between B3LYP and BHandH results, for the ESs relevant for the main OPA and TPA bands, with a few exceptions. As expected, one observes a somewhat larger delocalization within $\mathrm{BHandH}$, particularly visible on corresponding holes. However, the few exceptions confirm that the optimal fraction of exchange is a system-dependent parameter. ${ }^{54}$ Even so, B3LYP results suffer from a certain amount of inaccuracy, we believe that the main trends aimed to be discussed in the present work are trustable. For the sake of 
consistency, we will stick to B3LYP throughout the manuscript while BHandH results will be given as Supporting Information. We emphasis that comparison between nd and ndi series should not concern absolute amplitudes and all other approximations, namely fixed linewidth, limited number of ESs and absence of solvent and vibrational contributions should always been kept in mind, especially for comparison to experimental data.

\section{Molecular geometry}

When experiments are performed in solution at room temperature, various molecular conformations coexist leading to inhomogeneous broadening of optical spectra. Even if expected symmetry is not strictly retained, reminiscence of symmetry elements, such as centrosymmetry for quadrupoles, ${ }^{70} \quad \mathrm{C}_{3}{ }^{47}$ or $\mathrm{T}^{43}$ symmetry for three- or four-branched chromophores, are still observed in experimental findings and are often very useful to support interpretation. In addition, the amount of possible conformations increases with the number of monomeric units building up the multibranched molecule of interest. For these reasons, we did not perform an exhaustive review of attainable molecular arrangements. For the chromophores studied in the present work, the main conformational degree of freedom concerns torsional angles and the sequence of corresponding signs. Explored geometries showed energy differences small as compared to kT at room temperature. End-groups may either be approximately coplanar or perpendicular to the molecular mean plane (Figures 2, S1, S2). This may modify the state dipole moment, both in amplitude and vector components. But, it remains small enough to keep all chromophores in the so called neutral class of chromophores experiencing none or little solvatochromic behavior of their absorption spectra. ${ }^{42,71-73}$ For $\mathbf{2 d}$ compounds, we retained the conformation closest to inversion symmetry (Figure 2). For 3d compounds, the central triphenylamine (TPAmine) or triphenylbenzene (TPB) cores allow for either left or right handed skewness of the propeller core. Successive twist angles have been selected to ensure $\mathrm{C}_{3}$ symmetry and approximate planar geometries (Figure 2). The case of $4 \mathrm{de}$ and nde chromophores is somewhat more involved. First, chiral tetrahedral symmetry cannot be satisfied due to the geometry of the molecular branches. Among the carbon cored tetraphenyl analogs reported in the Cambridge Structural Data Base, ${ }^{74}$ some have almost $\mathrm{D}_{2}$ symmetry with phenyls related two by two like two butterflies, ${ }^{75}$ other have two by two almost perpendicular branches. ${ }^{76}$ For the present study, we retained the latter type of conformation (Figure 2) which also approximates tetrahedral symmetry well enough to lead to quasi-degeneracy of the three first ESs. ${ }^{43}$

The ground state optimized geometries of the branched structures show geometrical parameters for each branch quasi-identical to those of their dipolar counterparts. Twist angles around BP, TPAmine or TPB cores are all of about $45^{\circ}$, in line with those reported for related

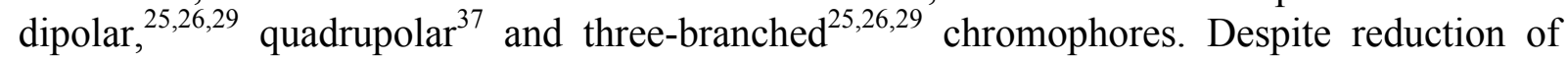
conjugation, as a result of the large twist angles, it has been demonstrated that significant electron communications can still be in action and can lead to enhanced NLO responses. ${ }^{17}$ The triazole moiety introduces additional twist angles along the molecular backbone which are significantly larger within ndi series than for members of nd series with a larger torsion for the phenyl ring connected to the nitrogen atom of the triazole ring (Table 2). The later is of about $42^{\circ}$ for members of ndi series while it is of about $30^{\circ}$ for compounds of nd series. The later is located in the middle of nd's molecular branches and thus favors electronic decoupling of both molecular ends. This may accentuate the CT problem discussed in the previous section. The twist with the phenyl ring connected to the carbon atom is much smaller ranging between 1 and $12^{\circ}$ (Table 2). These findings are consistent with the structural parameters of related triazole based structures reported in the Cambridge Structural Data Base. ${ }^{74}$ 


\section{One Photon Absorption (OPA)}

\section{nd series}

Given the $0.3 \mathrm{eV}$ red shift observed between available experimental data and calculated transition energies (Table 1), all members of nd series are predicted to show good transparency in the visible region (Figure 3). The first transition has a strong CT character, somewhat overestimated within B3LYP, thus the overlap between electron and hole wavefunctions is small and results in a small oscillator strength (Figure 3). As visible from the respective NTOs (Figures 4-6, S7-S12), the nature of the partial CT is preserved all along the series and corresponds to $\mathrm{CT}$ from the triazole-phenyl- $\mathrm{NMe}_{2}$ end group to the opposite end (core in multibranched systems). As the chemical composition of the later changes along the series, the transition energy of the first band changes as well. This is not the case for the main contribution to absorption which is dominated by a (a few for multibranched systems) higher lying ES near $4.6 \mathrm{eV}$ for all members of nd series. NTOs illustrate the nice correspondence observed all along the series and the large wave-function overlap facilitating the large oscillator strength (Figure 6 and S11). It should be noticed that for multibranched compounds, the oscillator strength is shared between several ESs (Figure 5) which are not all reported in Figure 4 (4de) for the sake of conciseness (Figures S11, S12). As both hole and electron are located on the peripheral triazole-phenyl- $\mathrm{NMe}_{2}$ moiety, it is little influenced by chemical substitution on the other end, by the nature of the core or by interbranch interactions in multimers and stays around $270 \mathrm{~nm}$ all over nd series. Thus, transition energies are hardly affected by the other molecular extreme or branching center. It should be noticed that, in the case of quadrupolar series, conclusion is less straightforward regarding the nature of the relevant ES bearing the main OPA oscillator strength. Within B3LYP, ES $\mid 9>$ bears the largest oscillator strength but has both hole and electron located at the molecular center (Figure S7), while BHandH leads to bright state $\mid 5>$ with major weight on the molecular ends (Figure S7, similar to ESs $\mid 10>$ and $\mid 11>$ of 3db shown Figure 8), consistent with the above mentioned picture. Within B3LYP, ES $\mid 12>$ shows up at $4.68 \mathrm{eV}$ with comparable shape but is a dark state for symmetry reasons while ES $\mid 13>$ is a mixture with only a small amount of oscillator strength (Figure S7).

In addition, comparison of related dipolar chromophores (da with $\mathbf{d b}$ (Figure 3 ) and $\mathbf{d c}$ up to de (Figure S4)) shows a slight but systematic red shift and amplitude increase with increasing molecular size. This shows that the choice for the dipolar unit used to investigate efficiency of the branching strategy may be important. Normalized absorption spectra show an overall additive behavior upon branching with no major enhancement or weakening (Figures 3, S3).

\section{ndi series}

For all members of this series, the main contribution to the first absorption band arises from the first (first few for multibranched chromophores) ES which bears significant oscillator strength (Figure 3). Despite the expected red shift upon branching, all chromophores maintain excellent transparency in the visible region. Comparison to the related chromophores ${ }^{31}$ shows a systematic blue shift of the first ES but only a slight reduction of the corresponding oscillator strength. This decrease has to be related to the large twist angle introduced by the triazole moiety (Table 2). Close lying higher ESs bearing significant oscillator strength lead to broad absorption spectra. NTOs illustrate the nature of corresponding electron redistribution upon excitation. Interestingly, while hole and electron associated to the first ES of dai, dbi, ${ }^{31}$ 2dai $^{31}{ }^{31} \mathbf{3 d b i}$ and 4dei are very similar (Figure 4, 7 and S13), with a periphery to core electronic redistribution, those of $\mathbf{d f f}^{31}$ (Figure S9) and $\mathbf{3} \mathbf{d f i}{ }^{31}$ (Figure 5) are much different as they both involve the TPAmine core up to the triazole moiety. The later are quite similar to 
those reported for a three-branched structure built by gathering three quadrupolar chromophores connected via common nitrogen. ${ }^{37}$ In both cases, there is a competition between the donating TPAmine core and donating $\mathrm{NMe}_{2}$ end-groups resulting in a larger donating character for the former. Normalized absorption spectra shown Figure 3 illustrate the different behavior upon branching. Again, to discuss OPA amplitude enhancement the choice of the monomeric analog is crucial as can be seen from comparison of dai and dbi spectra (Figure 3). Using dbi as reference, both 2dai and 3dbi show significant enhancement of the first absorption band (Figure 3), due to significant delocalization across the branching cores (Figures 7, S13), while that of 4dei is almost additive. On the contrary, the normalized amplitude of 3dfi decreases (Figure 3 ) as a result of slightly reduced delocalization of both the hole and the electron of its first $\mathrm{ES}^{31}$ (Figure 5, S9).

\section{position isomerism}

Comparison between members of nd and ndi series shows quite a few dramatic changes directly related to position isomerism of the 1,2,3-triazole ring. Due to the inverted position of the triazole ring, compounds of nd series have a large twist angle in the middle of the molecular backbone which potentially may disrupt their electronic communication between both molecular ends (Figures 2, S1, S2, Table 2). As a consequence, NTOs associated to the first excited state are less delocalized over the molecular backbone and corresponding oscillator strength are smaller than what is observed within ndi series (Figures 4, 5). While for all ndi members the first ES contributes predominantly to the main absorption band, nd compounds have a first absorption band with reduced amplitude followed by a stronger band showing up at approximately the same position for all members of the series (Figure 3 ).

Further comparison requires splitting both series into two sub-assemblies. Let us first concentrate on chromophores that do not contain the TPAmine moiety. For all of them, the terminal $\mathrm{NMe}_{2}$ group acts as the donating unit while the opposite core or end group, which may extend up to the triazole moiety, has an electron withdrawing character. For these compounds, one observes a systematic red shift for nd members as compared to the ndi analogs, but all maintain transparency in the visible region (Figure 3). The situation is quite different for the TPAmine based chromophores as the donating characters of the different amino groups compete. First, despite of the two donating end-groups, neither df nor dfi can be assimilated to a quadrupole (Figure S9). In fact, the branches deviates significantly from quadrupoles as both the chemical difference between TPamine and $\mathrm{NMe}_{2}$ and the dissymmetry of the 1,2,3-triazole moiety significantly break molecular symmetry. Comparison of NTOs of df and 3df with those of dfi (Figure S9) and 3dfi (Figure 5), respectively, shows a nice correspondence and nicely illustrates the competition between the donating moieties. Indeed, one observes a clear correspondence between ES $\mid 1>(\mid 3>)$ of $\mathbf{d f}$ with ES $\mid 6>(\mid 1>)$ of dfi. The same holds when ESs $\mid 1>$ and $\mid 2>(\mid 10>$ and $\mid 11>)$ of 3 df are compared to $\mid 10>$ and $\mid 11>(\mid 1>$ and $\mid 2>)$ of 3 dfi. The slight differences especially visible on the triazole moiety are directly related to the amplitude of the torsional angles with neighboring phenyl rings. Given the CT problem encountered for ndf members, the small residual differences with ndfi transition energies after systematic blue shift of ndf ones does not allow to conclude about the sign of the shift induced by position isomerism.

We underline that none of these NTOs evidence a strong participation of the triazole ring as electron donating or withdrawing species, when compared to all other moieties building the chromophores. Its main effect is to allow for a significant blue shift as compared to the related dipolar, $^{25,26,29}$ quadrupolar $^{70}$ and three-branched ${ }^{26,27,34,37,77}$ chromophores and triggers the competition between similar end-groups while maintaining good OPA responses. These 
findings corroborate with what has been previously inferred for isomeric triazole based CT chromophores $^{6}$ and for hyperbranched poly(1,2,3-triazole)s. ${ }^{7}$ In fact, Tang et al. ${ }^{7}$ conclude that "there exist ground-state interaction between TPAmine and triazole moieties in the polymers and that the electronic communications are dictated by the regioisomeric structures" and Diederich et al. "1,2,3-triazole makes a particularly intriguing linker where the CT path is interrupted... CT can occur and may be even quite efficient depending on the substitution pattern... responsible for about 50nm range of transition energies and also affects strongly the absorption intensities".

Finally, despite the expected red shift upon branching, all chromophores investigated in the present work maintain excellent transparency in the visible region. These findings corroborate experimental data ${ }^{4,13,40,41}$ and confirm that these triazole based chromophores are good candidates for optical limiting in the visible region. ${ }^{4}$

\section{Two photon absorption (TPA)}

\section{nd series}

Given that our results for this series may be affected by the CT problem discussed above, let us first weight up to which extend analysis of TPA properties is reasonable on the B3LYP level of theory. Comparison to BHandH calculations shows that the ES at the origin of the first TPA band keeps the same number and corresponding NTOs have similar shapes (Figures S7-S10) except for df. In both type of calculations, higher lying ESs are responsible for the appearance of additional TPA band having larger TPA amplitude (Figures 8, S5, S6). The behavior upon branching regarding amplitudes is also consistent within the two levels of theories (Figures 8, S5). The main difference concerns transition energies and especially state splitting which prevents any inference about the strength of the interbranch coupling within the Frenkel excitonic scheme. Indeed, B3LYP would lead to coupling energies less then $0.02 \mathrm{eV}$ while BHandH gives up to $0.09 \mathrm{eV}$ for 3df (Figures S7-S10).

For all but df, the first TPA maximum is found where expected, i.e. at the energy of the first, second, third and fourth ES respectively for all other dipoles, two-branched, three-branched and four-branched compounds. This is consistent with the Frenkel exitonic picture where dipolar species are approximated by a single ES. The corresponding electronic redistribution involves a periphery $\left(\mathrm{NMe}_{2}\right)$ to core electronic redistribution (Figures 4, 5, 7, S7, S8). Interestingly, df has only a weak shoulder nearby of the first ES while the main contribution to the first TPA band arises from the third ES (Figures 8, S5, S9). This may be a consequence of a pseudo-quadrupolar behavior of df which bears to nitrogen based end-groups that compete. Further inference about the underlying electronic redistribution of relevant ESs is hindered by significant differences between NTOs obtained at B3LYP and BHandH level of theory (Figure S9). The case of 3df is less tricky (Figure 5) thanks to the additional dissymmetry introduced on the TPAmine core which is tri-functionalized in the multimer, in a similar way of what has been obtained for an analogous branched chromophore built by gathering three quadrupolar units through a TPamine core. ${ }^{37}$ Even larger TPA amplitudes are predicted at higher energies (Figures 8, S5) for all but 3db, 3de and 4de for which analysis is limited by the number of ESs accounted for in our calculations. Unfortunately, systematic interrelationship with given ES is difficult and no general feature emerges from inspection of NTOs of those which have been identified (Figure S9, S10). 
In the offing of investigating branching strategy, it is worthwhile to emphasize the crucial role played by the choice of the benchmark monomer. Whereas only slight variations of both amplitudes and band position occur for various substitution of the sp3 carbon branching center (going from dc to de, Figure S6), the differences are large when replacing the phenyl end-group of da by a BP moiety to built $\mathbf{d b}$ (Figure 8). BP moiety not only stabilizes the first ES but also leads to a two-fold increase of the corresponding TPA amplitude thanks to expansion of delocalization over the BP end-group (Figure 6).

Whereas an overall additive behavior was observed for OPA, the branching strategy shows to be efficient for TPA responses (Figure 8). Once more, the TPAmine based chromophores are the exceptions among the series as the amplitude of the main TPA bands reveal essentially an additive behavior. Enhancement and reduction in specific spectral region are also observed. The overall behavior is quite similar to what has been reported for a branched quadrupole ${ }^{37}$ but careful inspection of the relevant NTOs shows that the nature of the ES in question is much less modified by the branching center. There is a clear correspondence between ES $\mid 1>$ of df (Figure S9) with $\mid 3>$ of $3 \mathbf{d f}$ (Figure 5). Within this picture, the amplitude of the first TPA band of $3 \mathbf{d f}$ has to be compared to the shoulder near $1.8 \mathrm{eV}$ in the TPA spectra of df and we recover the large enhancement expected for TPAmine branched dipoles. ${ }^{25,26}$ Despite similarities between NTO of $\mid 3>$ of df (Figure S9) with $\mid 27>$ of 3df (Figure 5), the large blue shift of the later prompts to carefulness in further interpretation. On the contrary, the picture is simple for all other members of this series as the first TPA band of monomers can be safely attributed to ES $\mid 1>$ and that of multimers to what predicts the exciton model. The quadrupole 2da shows the largest enhancement (a factor of two with respect to db) thanks to through BP core communication (Figures 8, S7). 3db evidences much less enhancement consistently with the other TPBcore ${ }^{17}$ analogs for which functionalization in meta-positions inhibits interbranch conjugation (Figure 8, S8). It should be noticed that comparison to the benchmark chromophore bearing a TPB end-group instead of BP would most probably lead at best to additive and, at worst, to reduced TPA responses. ${ }^{29}$ The case of C-centered branched chromophores is quite surprising as little through bond cross-talk is expected. Surprisingly, we observe a large enhancement that increases with increasing number of branches from 2 de up to 4de (Figure 8). Unfortunately, without any reasonable estimate for the interbranch coupling it is hard to conclude about the underlying nature of the interbranch interactions that may be mainly electrostatic or involve coherent interbranch interaction.

\section{ndi series}

Let us start with the monomeric benchmark chromophores. For the sake of conciseness, we did not consider any of the monomer substituted by $\mathrm{sp}^{3}$ carbons. Results obtained on nd series reveal only slight modifications between da and $\mathrm{sp}^{3}$ carbon analogs, the later having properties in between those of $\mathbf{d a}$ and $\mathbf{d b}$ (Figure 8). Thus, dai and $\mathbf{d b i}$ will respectively be used as lower and upper limits for investigating the effect of branching in all branched chromophores missing TPAmine. Figure 8 reports calculated TPA spectra for the three monomers dai, dbi and dfi. Replacement of the phenyl end-group by either BP or TPAmine leads to a dramatic increase of the TPA response, with the same trends previously observed for respective OPA (Figure 3). These substitutions induce respectively significant red and slight blue shifts of the first TPA band. The later is quite surprising given the large red shift observed for the main OPA band (Figure 3). In fact, the first TPA band of both dai and dbi mainly involves ES $\mid 1>$ which also dominates the OPA spectra. On the contrary, that of dfi originates from ES $\mid 6>$ (Figure 8, S9), while ES $\mid 1>$ appears as a very slight shoulder on the low energy part of the spectrum. Comparison of corresponding NTOs is informative as ES $\mid 6>$ of dfi (Figure S9) shows similar periphery to core electronic redistribution to ES $\mid 1>$ of dai 
and dbi (Figure 7). Thus, while for the first OPA band the nature of the underlying electronic redistribution is completely different for dfi, it is not for the final TPA state giving rise to the first TPA band. Furthermore, the NTO of an electron for dfi is the same for ES $\mid 1>$ and ES $\mid 6>$ while the respective hole orbitals involve either TPAmine or $\mathrm{NMe}_{2}$ end-groups, indicating the competition between the two donating nitrogen based moieties (Figure S9). The second TPA maxima near $2.5 \mathrm{eV}$ (Figure 8) can be assigned to ESs $|5>| 6>$, and $\mid 13>$ respectively for dai, dbi (Figure 7) and dfi (Figure S9).

TPA spectra normalized for the number of branches, reported in Figure 8, reveal significant enhancement for all branched chromophores with coincident peak positions (shoulder for 4dei) for the first TPA band. Higher lying ESs lead to even larger TPA responses on the high energy part of the spectra (Figure 4, 7), except for 3dfi for which analysis is hindered by the limited number of ESs accounted for. For all branched chromophores but 3dfi, the first TPA maximum is found consistently with the Frenkel exciton model predictions: second, third and fourth ES for 2dai, 3dbi, 4dei respectively. The final ES involves a periphery $\left(\mathrm{NMe}_{2}\right)$ to core electronic redistribution (Figures $4,6, \mathrm{~S} 13$ ) that nicely compares with ES $\mid 1>$ of dai and dbi (Figure 7), especially for $\mathbf{3 d b i}$ and 4 dei. However, predicted state splitting with respect to benchmark monomers is not at all satisfied, and manifests itself by correspondence in band position of the first TPA bands of monomers and multimers. From the state splitting calculated for each multimer, estimates of the interbranch coupling lead to $0.02,0.03$ and $0.08 \mathrm{eV}$ for $\mathbf{3} \mathbf{d b i}$, 4dei and both $\mathbf{2 d a i}$ and $\mathbf{3 d f i}$, respectively. These findings correlate nicely with the shapes of corresponding NTO as hole and electrons have at least significant if not prevailing weight on the branching center for the last two chromophores. 3dbi evidences the lowest enhancement among the series as was the case for $\mathbf{3} \mathbf{d b}$ and the same reasoning holds. The case of C-centered branched chromophores 4dei is similar to that of $\mathbf{3} \mathbf{d b i}$ as the enhancement near $2.2 \mathrm{eV}$ partly results from the tail of the band located near $2.4 \mathrm{eV}$. The later can be assigned to a couple of higher lying excited states such as ES $\mid 27>$ (Figure 4) showing similitude to some degree with ESs $\mid 5>$ and $\mid 6>$ of dai and dbi, respectively (Figure 7). For 2dai, the strong through bond communication allowed by the BP core leads to a significant mixture of dipolar ESs (Figure S13) and two-fold enhancement of the normalized TPA amplitude at the first maximum as compared to dbi. ${ }^{31}$ Contrarily to the three other branched ndi compounds, but consistently to what has been observed for the benchmark chromophore dfi, no TPA maximum is visible in the vicinity of ES $\mid 3>$ of $3 \mathrm{dfi}$ (Figures 5,8). The main TPA band has to be related to ES $\mid 12>$, and the corresponding NTOs show a marked correspondence with those of ES $\mid 6>$ of $\mathrm{dfi}^{31}$ (Figures 8, S9). Thus, for all ndi chromophores the first TPA band corresponds to an ES with quasi identical holes and electrons, i.e. underlying electronic redistribution from periphery $\left(\mathrm{NMe}_{2}\right.$ side) to the core. Moreover, significant enhancement of the normalized TPA amplitude is also in action for 3dfi. Therefore, the branching strategy shows to be efficient for all four investigated cores. The main change occurring for TPAmine based chromophores is decoupling of OPA and TPA states leading to a consequent blue shift of the first TPA maximum which shows the largest values among both monomers and multimers. Among the reasons for large TPA amplitudes, we notice that higher lying ESs open the possibility of manifold coupling to the intermediate lower lying ESs which have sizeable transition dipole moments.

\section{position isomerism}

The overall effects of position isomerism of the 1,2,3-triazole ring on TPA responses are less dramatic then those reported for OPA responses, except for TPAmine based chromophores. In fact, ESs at the origin of the main OPA band are quite different for the two series but this is not the case for the first TPA band. Consistently with the Frenkel exciton picture, where 
monomers are reduced to a single ES (being the first ES) large TPA responses originate from the first, second, third and fourth ESs, respectively, for monomers, two-, three- and fourbranched compounds, for both position isomers. In addition, NTOs of the final ES show comparable periphery (NMe2 end-groups) to core electron redistribution for all. Overall, their delocalization over the molecular branches is significantly larger for ndi than nd, even so comparison is limited due to the occurrence of CT problems for nd compounds. Accordingly, transition dipole moments are significantly enhanced for ndi chromophores with respect to their nd analogs and so are the related TPA responses.

The ranking of TPA amplitudes and relative peak positions among the dipoles stays the same for both position isomers. Substitution of the phenyl ring by a BP moiety leads to noteworthy increase of TPA amplitudes and red shifts. Further substitution by TPamine core leads to an additional TPA enhancement but with a significant red shift of the first TPA band.

Interestingly, none of the investigated branched chromophores showed any quantitative agreement with the Frenkel exciton model such as band splitting around the first ES of the benchmark monomer. TPA spectra of nd chromophores undergo significant red shifts with respect to their monomeric counterpart, while the first TPA maximum of each ndi chromophore shows up almost at the same energy as its dipolar benchmark. On the contrary, the behavior upon branching is quite similar in both series with moderate enhancement for TPB core, sizeable enhancement for $\mathrm{sp}^{3}$ carbon core, end two-fold increase for BP core. Thus, combining the branching strategy with inversion of the triazole moiety allows for a spectral tuning of TPA responses to a larger extent than OPA responses, while maintaining the efficiency of cooperative effects.

The different behavior upon triazole inversion in TPamine based chromophores is directly related to the presence of two donating units instead of one. As already mentioned for investigation of their OPA responses, the triazole moiety controls competition between the two amino groups. This is nicely illustrated by NTOs of relevant ESs. Indeed, there is a clear correspondence between NTOs of ESs $\mid 3>$ of $\mathbf{3 d f}$ and $\mid 12>$ of $3 \mathbf{d f i}$ (Figure 5). These final ESs contribute predominantly to the first TPA band. They involve periphery to core electronic redistribution comparable to what was found for the compounds missing the TPAmine moiety. The same conclusions hold for ESs $\mid 1>$ of df and $\mid 6>$ of dfi (Figure S9). Moreover, NTOs of ESs $\mid 12>$ of $\mathbf{3 d f}$ and $\mid 3>$ of $\mathbf{3 d f i}$ (Figure 5) have also very similar shapes and are thus consistent with the picture of competing end groups. Corresponding electronic redistributions being localized on the molecular center, they do not contribute strongly to the TPA responses. Thus, the overall competition between the donating end groups explains why both dfi and $3 \mathbf{d f i}$ have their first TPA maximum far blue shifted from the position expected from a simple reasoning. 


\section{IV.Conclusion}

In conclusion, our quantum-chemical study based on TD-DFT methodology reveals that introduction of triazole leads to dramatic changes in optical properties of chromophores especially for multibranched systems. The triazole ring substantially modifies electronic communication and delocalization between the electron donating end-groups and the branching center via torsion between phenyl and triazole rings (which modifies piconjugation) as well as through its position isomerism. This adds to the multibranching interactions allowing for stark and intuitively unexpected modifications of the excited state structure and, subsequently, relevant linear and nonlinear optical responses. In particular, when compared to the related analogs, introduction of the triazole moiety in the molecular backbone allows for an overall blue shift of both OPA and TPA bands with a moderate reduction of their response amplitudes. Their ability to be promising candidates for optical limiting in the visible region ${ }^{4,13}$ is thus confirmed for a diversified set of chromophores. In addition, larger TPA responses are predicted at higher energies due to contribution from highlying ESs, as has been found for similar chromophores. ${ }^{37}$

The changes observed between the two investigated 1,2,3-triazole regioisomers can be related to differences in the amplitude of the torsional angles with neighboring phenyl rings. The twist angle with the phenyl substituted at the nitrogen position is significantly larger then that of phenyl attached to the carbon atom. The former is located in the middle of nd's molecular branch(es), thus favoring electronic decoupling of both molecular ends. Besides the overall blue shift when going from nd series to ndi, this electronic decoupling increases the CT character of the first absorption band and consequently decreases it's OPA oscillator strength. The main absorption band shows up near $4.6 \mathrm{eV}$ independently of the nd chromophore of interest. This is a direct consequence of hole and electron localization on the peripheral $\mathrm{NMe}_{2}$ donating moiety that ensures little influence on the relevant ES(s) of chemical substitution on the other end, including nature of the core or interbranch interactions in multimers. Position isomerism has less impact on TPA properties except for TPAmine based chromophores. The later bear nitrogen based donating moieties on both molecular ends or periphery and core for monomers and three-branched derivatives, respectively. Thus, monomers could be thought as pseudo-quadrupoles but a detailed inspection of NTOs reveals a more subtle behavior. In fact, the respective role of TPAmine and NMe2 moieties is interchanged and triggered by the triazole moiety. Calculations show that the nature of the electronic redistribution (periphery to TPAmine core) associated to the first TPA band of the three-branched compounds is preserved. As a consequence, while the main contribution to this TPA band arises from the third ES for 3df, it corresponds to the twelve's ES for $\mathbf{3 d f i}$. In addition, none of the monomers has its first TPA band in the vicinity of the first ES. Thus, the apparent behavior upon branching is dramatically different for both isomers with a large red shift and almost additive behavior for $\mathbf{3} \mathbf{d f}$ as compared to $\mathbf{d f}$ and concomitance and enhancement for $\mathbf{3 d f i}$ as compared to dfi. This intricate finding renders the Frenkel exciton model useless for these TPAmine based chromophores.

For all other chromophores, results are qualitatively consistent with the Frenkel exciton picture as the main TPA band can be associated to the first, second, third and fourth ESs respectively for dipoles, quadrupoles, three- and four-branched derivatives. As found for related chromophores, the donating end-groups confer an electron withdrawing character to the $\mathrm{BP}^{37}, \mathrm{TPB}^{29}$ and $\mathrm{sp}^{3}$ carbon moieties and monomers have a significant dipolar character. The branching strategy shows to be efficient as sizeable enhancement of TPA responses are 
observed with moderate effect for TPB core and about twofold enhancement for both BP core and carbon-cored tetramers. However, we underline that the amount of enhancement strongly depends on the choice of the benchmark monomer. In addition, for large chromophores, the increasing number of conformational degrees of freedom may lead to experimentally broadened and reduced TPA responses when performed in solutions at room temperature, as a result of oscillator strength redistribution (intra monomer) and inhomogeneous broadening.

These results and further inspection of NTOs clearly show that the 1,2,3-triazole moiety has neither an electron donating nor an electron withdrawing character. This corroborates earlier findings where triazole has been described as an intriguing linker that introduces conjugation disruption while maintaining significant electronic communication and optical properties dictated by the regiosiomeric structure. This subtle-acting of the triazole moiety may be combined to complementary routes of molecular engineering. Replacement of donating endgroups by electron-withdrawing ones should lead to further blue shifts that may allow to keep full transparency in the visible region for even larger branched systems. ${ }^{25,26,29}$ Substitution of the BP core by a fluorene core should allow for increased conjugation along the quadrupolar backbone and both improve OPA and TPA responses. ${ }^{37}$ Slight dissymmetrical functionalization could also offer interesting perspectives for spatial control of the fluorescence properties while maintaining sizeable TPA responses. ${ }^{63}$

From the theoretical perspectives, these series are also a kind of tutorial example of isomeric compounds that are quite sensitive to the specific exchange-correlation DFT functional in use. In fact, while description of one of the isomers shows very good agreement with experimental results, e.g. for transition energies and oscillator strength, the other is quite poorly described with the very same functional. In fact, besides comparison to available experimental data, in the B3LYP model the first ESs of nd members have small integral overlap between their electron and hole orbitals, which renders these states to be optically weak. This small overlap between NTO orbitals of ES indicates its CT nature (in a general sense) and points to a possible overestimated red-shift of the transition energy in semi-local DFT models. Nevertheless, increase of the amount of exact exchange (going from B3LYP to BHandH) allows for at least qualitative interpretation of the optical responses of the studied chromophores. The present example illustrates the need of further improvements in developing better XC functional as it shows that increased amount of exact exchange improves respective oscillator strengths of different electronic transitions while at the same time the respective transition energies become over estimated.

Acknowledgement. We wish to thank Dr. Olivier Mongin and Manuel Parent for stimulating discussions. This work was performed in part at the US Department of Energy, Center for Integrated Nanotechnologies (CINT), at Los Alamos National Laboratory (LANL) (contract DE-AC52-06NA25396). This work was granted access to the HPC resources of CINES under the allocation 2005-[c20050822414] and 2008-[x20080825087] made by GENCI (Grand Equipement National de Calcul Intensif).

Supporting Information Available: Table of spectral linewidths, additional calculated OPA and TPA spectra, additional calculated NTOs and corresponding transition energies, transition wavelengths and oscillator strengths. This material is available free of charge via the Internet at http://pub.acs.org.. 


\section{References}

(1) Kolb, H. C.; Finn, M. G.; Sharpless, K. B. Angewandte Chemie International Edition 2001, 40, 2004.

(2) Qin, A.; Lam, J. W. Y.; Tang, B. Z. Chemical Society Reviews, 2010.

(3) Zhou, Z.; Fahrni, Journal of the American Chemical Society 2004, 126, 8862.

(4) Parent, M.; Mongin, O.; Kamada, K.; Katan, C.; Blanchard-Desce, M. Chemical Communications 2005, 2029.

(5) Qin, A.; Jim, C. K. W.; Lu, W.; Lam, J. W. Y.; Häussler, M.; Dong, Y.; Sung, H. H. Y.; Williams, I. D.; Wong, G. K. L.; Tang, B. Z. Macromolecules 2007, 40, 2308.

(6) Jarowski, P. D.; Wu, Y.-L.; Schweizer, W. B.; Diederich, F. Org. Lett. 2008, 10, 3347.

(7) Qin, A.; Lam, J. W. Y.; Jim, C. K. W.; Zhang, L.; Yan, J.; Häussler, M.; Liu, J.; Dong, Y.; Liang, D.; Chen, E.; Jia, G.; Tang, B. Z. Macromolecules 2008, 41, 3808.

(8) Meudtner, Robert M.; Ostermeier, M.; Goddard, R.; Limberg, C.; Hecht, S. Chemistry - A European Journal 2007, 13, 9834.

(9) Schweinfurth, D.; Hardcastle, K. I.; Bunz, U. H. F. Chem. Commun. 2008, 2203.

(10) Li, Z. a.; Yu, G.; Wu, W.; Liu, Y.; Ye, C.; Qin, J.; Li, Z. Macromolecules 2009, 42, 3864.

(11) Li, Z. a.; Yu, G.; Hu, P.; Ye, C.; Liu, Y.; Qin, J.; Li, Z. Macromolecules 2009, 42, 1589.

(12) Xie, J.; Hu, L.; Shi, W.; Deng, X.; Cao, Z.; Shen, Q. Journal of Polymer Science Part B: Polymer Physics 2008, 46, 1140.

(13) Zoon, P. D.; Stokkum, I. H. M. v.; Parent, M.; Mongin, O.; Blanchard-Desce, M.; Brouwer, A. M. Physical Chemistry Chemical Physics 2010, 12, 2706.

(14) Fréchet, J. M. J. Proceedings of the National Academy of Sciences of the United States of America 2002, 99, 4782.

(15) Sullivan, P. A.; Rommel, H.; Liao, Y.; Olbricht, B. C.; Akelaitis, A. J. P.; Firestone, K. A.; Kang, J.-W.; Luo, J.; Davies, J. A.; Choi, D. H.; Eichinger, B. E.; Reid, P. J.; Chen, A.; Jen, A. K. Y.; Robinson, B. H.; Dalton, L. R. Journal of the American Chemical Society 2007, 129, 7523.

(16) He, G. S.; Tan, L. S.; Zheng, Q.; Prasad, P. N. Chemical Reviews 2008, 108, 1245.

(17) Terenziani, F.; Katan, C.; Badaeva, E.; Tretiak, S.; Blanchard-Desce, M. Advanced Materials 2008, 20, 4641.

(18) Rumi, M.; Barlow, S.; Wang, J.; Perry, J. W.; Marder, S. R. In Photoresponsive Polymers I; Advances in Polymer Science, Heidelberg, S. B., Ed. 2008; Vol. 213, p 1.

(19) Pawlicki, M.; Collins, H. A.; Denning, R. G.; Anderson, H. L. Angewandte Chemie-International Edition 2009, 48, 3244.

(20) Specht, A.; Bolze, F.; Omran, Z.; Nicoud, J.-F.; Goeldner, M. HFSP Journal 2009, $3,255$.

(21) Kim, H. M.; Cho, B. R. Chemical Communications 2009, 153.

(22) Joshi, M. P.; Swiatkiewicz, J.; Xu, F. M.; Prasad, P. N. Optics Letters 1998, 23, 1742.

(23) Chung, S. J.; Kim, K. S.; Lin, T. H.; He, G. S.; Swiatkiewicz, J.; Prasad, P. N. Journal of Physical Chemistry B 1999, 103, 10741.

(24) Cho, B. R.; Son, K. H.; Lee, S. H.; Song, Y. S.; Lee, Y. K.; Jeon, S. J.; Choi, J. H.; 
(25) Katan, C.; Terenziani, F.; Mongin, O.; Werts, M. H. V.; Porres, L.; Pons, T.;

Mertz, J.; Tretiak, S.; Blanchard-Desce, M. Journal of Physical Chemistry A 2005, 109, 3024.

(26) Katan, C.; Terenziani, F.; Droumaguet, C. L.; Mongin, O.; Werts, M. H. V.; Tretiak, S.; Blanchard-Desce, M.; Manfred, E., Ed.; SPIE: 2005; Vol. 5935, p 593503.

(27) Terenziani, F.; Morone, M.; Gmouh, S.; Blanchard-Desce, M. ChemPhysChem 2006, 7, 685 .

(28) Allain, C.; Schmidt, F.; Lartia, R.; Bordeau, G.; Fiorini-Debuisschert, C.; Charra, F.; Tauc, P.; Teulade-Fichou, M.-P. ChemBioChem 2007, 8, 424.

(29) Terenziani, F.; Le Droumaguet, C.; Katan, C.; Mongin, O.; Blanchard-Desce, M. Chemphyschem 2007, 8, 723.

(30) Kim, H. M.; Seo, M. S.; Jeon, S.-J.; Cho, B. R. Chemical Communications 2009, 7422.

(31) Katan, C.; Tretiak, S.; Even, J. In Nanophotonics III; 1 ed.; SPIE: 2010; Vol. 7712, p 77123D.

(32) Drobizhev, M.; Karotki, A.; Dzenis, Y.; Rebane, A.; Suo, Z. Y.; Spangler, C. W. Journal of Physical Chemistry B 2003, 107, 7540.

(33) Yoo, J.; Yang, S. K.; Jeong, M. Y.; Ahn, H. C.; Jeon, S. J.; Cho, B. R. Organic Letters 2003, 5, 645.

(34) Mongin, O.; Porres, L.; Katan, C.; Pons, T.; Mertz, J.; Blanchard-Desce, M. Tetrahedron Letters 2003, 44, 8121.

(35) Bartholomew, G. P.; Rumi, M.; Pond, S. J. K.; Perry, J. W.; Tretiak, S.; Bazan, G. C. Journal of the American Chemical Society 2004, 126, 11529.

(36) Varnavski, O.; Yan, X. Z.; Mongin, O.; Blanchard-Desce, M.; Goodson, T. Journal of Physical Chemistry C 2007, 111, 149.

(37) Katan, C.; Tretiak, S.; Werts, M. H. V.; Bain, A. J.; Marsh, R. J.; Leonczek, N.; Nicolaou, N.; Badaeva, E.; Mongin, O.; Blanchard-Desce, M. Journal of Physical Chemistry B 2007, 111, 9468.

(38) Rumi, M.; Pond, S. J. K.; Meyer-Friedrichsen, T.; Zhang, Q.; Bishop, M.; Zhang, Y.; Barlow, S.; Marder, S. R.; Perry, J. W. 2008 Journal of Physical Chemistry C 112,8061 .

(39) Varnavski, O. P.; Ostrowski, J. C.; Sukhomlinova, L.; Twieg, R. J.; Bazan, G. C.; Goodson, T. Journal of the American Chemical Society 2002, 124, 1736.

(40) Parent, M. Ph.D. Thesis, University of Rennes 2008.

(41) Blanchard-Desce and coll. Unpublished.

(42) Terenziani, F.; Painelli, A.; Katan, C.; Charlot, M.; Blanchard-Desce, M. Journal of the American Chemical Society 2006, 128, 15742.

(43) Lambert, C.; Schmalzlin, E.; Meerholz, K.; Brauchle, C. Chemistry-a European Journal 1998, 4, 512.

(44) Tretiak, S.; Chernyak, V.; Mukamel, S. Journal of Physical Chemistry B 1998, $102,3310$.

(45) Poliakov, E. Y.; Chernyak, V.; Tretiak, S.; Mukamel, S. The Journal of Chemical Physics 1999, 110, 8161.

(46) Chernyak, V.; Poliakov, E. Y.; Tretiak, S.; Mukamel, S. The Journal of Chemical Physics 1999, 111, 4158.

(47) Beljonne, D.; Wenseleers, W.; Zojer, E.; Shuai, Z. G.; Vogel, H.; Pond, S. J. K.; Perry, J. W.; Marder, S. R.; Bredas, J. L. Advanced Functional Materials 2002, 12, 631. 
(48) Casida, M. E.; Jamorski, C.; Casida, K. C.; Salahub, D. R. Journal of Chemical Physics 1998, 108, 4439.

(49) Furche, F.; Ahlrichs, R. Journal of Chemical Physics 2002, 117, 7433.

(50) Grimme, S. In Reviews in Computational Chemistry 2004; Vol. 20, p 153.

(51) Dreuw, A.; Head-Gordon, M. Chemical Reviews 2005, 105, 4009.

(52) Champagne, B.; Guillaume, M.; Zutterman, F. Chemical Physics Letters 2006, $425,105$.

(53) Miura, M.; Aoki, Y.; Champagne, B. The Journal of Chemical Physics 2007, 127, 084103.

(54) Magyar, R. J.; Tretiak, S. Journal of Chemical Theory and Computation 2007, 3, 976.

(55) Jacquemin, D.; Wathelet, V.; Perpète, E. A.; Adamo, C. Journal of Chemical Theory and Computation 2009, 5, 2420.

(56) Tretiak, S.; Chernyak, V. Journal of Chemical Physics 2003, 119, 8809.

(57) Masunov, A.; Tretiak, S. The Journal of Physical Chemistry B 2003, 108, 899.

(58) Kobko, N.; Masunov, A.; Tretiak, S. Chemical Physics Letters 2004, 392, 444.

(59) Kauffman, J. F.; Turner, J. M.; Alabugin, I. V.; Breiner, B.; Kovalenko, S. V.; Badaeva, E. A.; Masunov, A.; Tretiak, S. The Journal of Physical Chemistry A 2005, 110, 241.

(60) Badaeva, E. A.; Timofeeva, T. V.; Masunov, A.; Tretiak, S. The Journal of Physical Chemistry A 2005, 109, 7276.

(61) Clark, A. E. The Journal of Physical Chemistry A 2006, 110, 3790.

(62) Wu, C.; Tretiak, S.; Chernyak, V. Y. Chemical Physics Letters 2007, 433, 305.

(63) Katan, C.; Charlot, M.; Mongin, O.; Le Droumaguet, C. 1.; Jouikov, V.; Terenziani, F.; Badaeva, E.; Tretiak, S.; Blanchard-Desce, M. The Journal of Physical Chemistry B 2010, 114, 3152.

(64) Cross-shaped chromophores consisting of four donor substituted branches linked to an aromatic core have already been investigated but show significant differences, e.g. in OPA spectra, and can well be described as a dimer of corresponding quadrupolar benchmarks. ${ }^{38}$

(65) Ultrafast fluorescence spectroscopy has already been performed on a related carbon-cored tetramer. ${ }^{39}$

(66) Frisch, M. J.; Trucks, G. W.; Schlegel, H. B., Scuseria; G. E., Robb, M. A.; Cheeseman, J. R.; Montgomery Jr., J. A.; Stratmann, R. E.; Burant, J. C.; Dapprich, S.; Millam, J. M.; Daniels, A. D.; Kudin, K. N.; Strain, M. C.; Farkas, O.; Tomasi, J.; Barone, V.; Cossi, M.; Cammi, R.; Mennucci, B.; Pomelli, C.; Adamo, C.; Clifford, S.; Ochterski, J.; Petersson, G. A.; Ayala, P. Y.; Cui, Q.; Morokuma, K.; Malick, D. K.; Rabuck, A. D.; Raghavachari, K.; Foresman, J. B.; Cioslowski, J.; Ortiz, J. V.; Stefanov, B. B.; Liu, G.; Liashenko, A.; Piskorz, P.; Komaromi, I.; Gomperts, R.; Martin, R. L.; Fox, D. J.; Keith, T.; Al-Laham, M. A.; Peng, C. Y.; Nanayakkara, A.; Gonzalez, C.; Challacombe, M.; Gill, P. M. W.; Johnson, B.; Chen, W.; Wong, M. W.; Andres, J. L.; Head-Gordon, M.; S.; R. E.; Pople, J. A. Gaussian 98, Revision A.11, Gaussian, Inc., Pittsburgh PA 2001.

(67) Frisch, M. J.; Trucks, G. W.; Schlegel, H. B.; Scuseria, G. E.; Robb, M. A.; Cheeseman, J. R.; Montgomery, Jr.; J. A.; Vreven, T.; Kudin, K. N.; Burant, J. C.; Millam, J. M.; Iyengar, S. S.; Tomasi, J.; Barone, V.; Mennucci, B.; Cossi, M.; Scalmani, G.; Rega, N.; Petersson, G. A.; Nakatsuji, H.; Hada, M.; Ehara, M.; Toyota, K.; Fukuda, R.; Hasegawa, J.; Ishida, M.; Nakajima, T.; Honda, Y.; Kitao, O.; Nakai, H.; Klene, M.; Li, X.; Knox, J. E.; Hratchian, H. P.; Cross, J. B.; Bakken, V.; Adamo, C.; Jaramillo, J.; Gomperts, R.; Stratmann, R. E.; Yazyev, O.; 
Austin, A. J.; Cammi, R.; Pomelli, C.; Ochterski, J. W.; Ayala, P. Y.; Morokuma, K.; Voth, G. A.; Salvador, P.; Dannenberg, J. J.; Zakrzewski, V. G.; Dapprich, S.; Daniels, A. D.; Strain, M. C.; Farkas, O.; Malick, D. K.; Rabuck, A. D.; Raghavachari, K.; Foresman, J. B.; Ortiz, J. V.; Cui, Q.; Baboul, A. G.; Clifford, S.; Cioslowski, J.; Stefanov, B. B.; Liu, G.; Liashenko, A.; Piskorz, P.; Komaromi, I.; Martin, R. L.; Fox, D. J.; Keith, T.; Al-Laham, M. A.; Peng, C. Y.; Nanayakkara, A.; Challacombe, M.; Gill, P. M. W.; Johnson, B.; Chen, W.; Wong, M. W.; Gonzalez, C.; Pople, J. A. Gaussian 03, Revision D.02, Gaussian, Inc.: Wallingford CT 2004.

(68) Martin, R. L. Journal of Chemical Physics 2003, 118, 4775.

(69) Kokalj, A. Computational Materials Science 2003, 28, 155.

(70) Mongin, O.; Porres, L.; Charlot, M.; Katan, C.; Blanchard-Desce, M. Chemistry-a European Journal 2007, 13, 1481.

(71) Lu, D.; Chen, G.; Perry, J. W.; Goddard, W. A. Journal of the American Chemical Society 1994, 116, 10679.

(72) Barzoukas, M.; Blanchard-Desce, M. J. Chem. Phys. 2000, 113, 3951.

(73) Terenziani, F.; Sissa, C.; Painelli, A. Journal of Physical Chemistry B 2008, 112, 5079 .

(74) Allen, F. H. Acta Crystallographica 2002, 58, 380.

(75) Basavoju, S.; Aitipamula, S.; Desiraju, G. R. CrystEngComm, 6, 120.

(76) Oldham, W. J.; Lachicotte, R. J.; Bazan, G. C. Journal of the American Chemical Society 1998, 120, 2987.

(77) Porres, L.; Mongin, O.; Katan, C.; Charlot, M.; Pons, T.; Mertz, J.; BlanchardDesce, M. Organic Letters 2004, 6, 47. 
J. Chem. Theory Comput., 2010, 6 (11), pp 3410-3426 ; DOI: 10.1021/ct1004406

Table 1. Calculated one-photon vertical absorption maxima at the TD-B3LYP/6-31G//HF/6-31G level in vacuum and experimental one-photon absorption maxima in toluene. , $13,40^{\text {In }}$ parentheses, corresponding transition energies in $\mathrm{eV}$.

\begin{tabular}{|c|c|c|c|c|c|c|c|c|c|c|}
\hline & \multicolumn{2}{|c|}{$\mathbf{2 d a}$} & \multicolumn{2}{c|}{ 3db } & dai & dbi & 2dai & 3dbi & 3dfi & 4dei \\
\hline$\lambda^{\text {calc }} \mathrm{nm}$ & 388 & 280 & 381 & 274 & 286 & $299^{\mathrm{a}}$ & $313^{\mathrm{a}}$ & 303 & $348^{\mathrm{a}}$ & 292 \\
$(\mathrm{eV})$ & $(3,19)$ & $(4,42)$ & $(3,25)$ & $(4,52)$ & $(4,34)$ & $(4,15)$ & $(3,96)$ & $(4,09)$ & $(3,56)$ & $(4,25)$ \\
\hline$\lambda^{e x p} \mathrm{~nm}$ & $350^{\mathrm{b}}$ & $296^{\mathrm{b}}$ & $347^{\mathrm{b}}$ & $293^{\mathrm{b}}$ & $303^{\mathrm{b}}$ & $310^{\mathrm{c}}$ & $325^{\mathrm{c}, \mathrm{d}}$ & $317^{\mathrm{b}}$ & $347^{\mathrm{d}}$ & $311^{\mathrm{b}}$ \\
$(\mathrm{eV})$ & $(3,54)$ & $(4,19)$ & $(3,57)$ & $(4,23)$ & $(4,09)$ & $(4,00)$ & $(3,82)$ & $(3,91)$ & $(3,57)$ & $(3,99)$ \\
\hline
\end{tabular}

${ }^{\mathrm{a}}$ from ref. $31^{\mathrm{b}}$ from ref. $40^{\mathrm{c}}$ from ref. $13^{\mathrm{d}}$ from ref. 4

Table 2. Average torsional angles in degrees between phenyl and triazole rings.

\begin{tabular}{|c|c|c|c|c|c|c|c|c|c|c|c|c|c|c|c|}
\hline $\begin{array}{l}\text { attachment } \\
\text { point }\end{array}$ & da & db & de & df & 2da & $3 \mathrm{db}$ & $3 d f$ & $4 \mathrm{de}$ & dai & dbi & dfi & 2dai & $3 \mathrm{dbi}$ & $3 \mathrm{dfi}$ & 4dei \\
\hline $\mathbf{N}$ & 31 & 31 & 30 & 34 & 30 & 30 & 33 & 28 & 42 & 42 & 42 & 42 & 42 & 42 & 42 \\
\hline C & 2 & 2 & 3 & 3 & 2 & 2 & 2 & 3 & 1 & 0 & 2 & 3 & 5 & 9 & 7 \\
\hline
\end{tabular}


J. Chem. Theory Comput., 2010, 6 (11), pp 3410-3426 ; DOI: 10.1021/ct1004406

nd

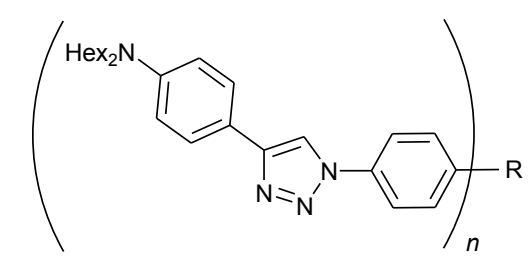

da: $\quad n=1, \mathrm{R}=\mathrm{H}$

db: $\quad n=1, \mathrm{R}=\mathrm{C}_{6} \mathrm{H}_{5}$

dc: $\quad n=1, \mathrm{R}=\mathrm{CH}_{3}$

dd: $\quad n=1, \mathrm{R}=\mathrm{C}\left(\mathrm{CH}_{3}\right)_{3}$

de: $n=1, \mathrm{R}=\mathrm{C}\left(\mathrm{C}_{6} \mathrm{H}_{5}\right)_{3}$

df: $\quad n=1, \mathrm{R}=\mathrm{N}\left(\mathrm{C}_{6} \mathrm{H}_{5}\right)_{2}$

2da: $n=2, \mathrm{R}=$ -

2de: $n=2, \mathrm{R}=\mathrm{C}\left(\mathrm{C}_{6} \mathrm{H}_{5}\right)_{2}$

3db: $\quad n=3, \mathrm{R}=1,3,5-\mathrm{C}_{6} \mathrm{H}_{3}$

3de: $n=3, \mathrm{R}=\mathrm{C}\left(\mathrm{C}_{6} \mathrm{H}_{5}\right)$

3df: $n=3, \mathrm{R}=\mathrm{N}$

4de: $n=4, \mathrm{R}=\mathrm{C}$ ndi

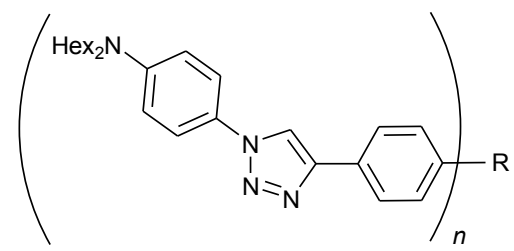

dai

dbi

dfi

2dai

3dbi

3dfi

4dei

Figure 1. Molecular structures of 1,2,3-triazole based nd chromophores (Left) and corresponding ndi position isomers (Right). Hexyl solubilizing chains have been replaced by methyl groups.

de
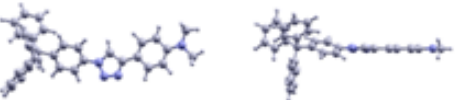

$2 \mathrm{da}$
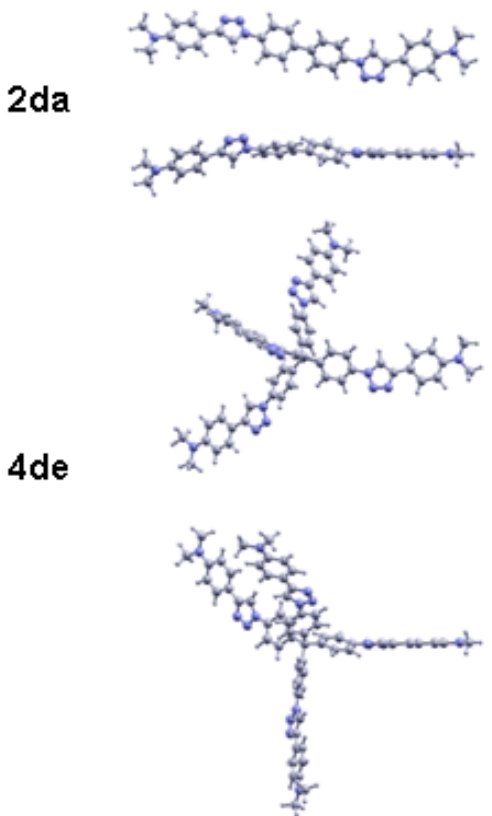

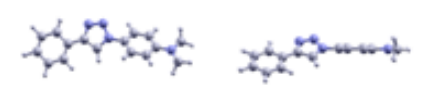

dai

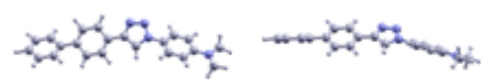

dbi

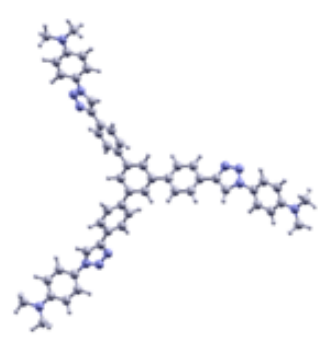

3dbi

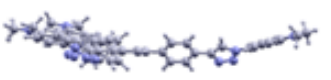

Figure 2. Optimized ground state molecular geometries at HF/6-31G level of theory for selected nd (Left) and ndi (Right) chromophores. 


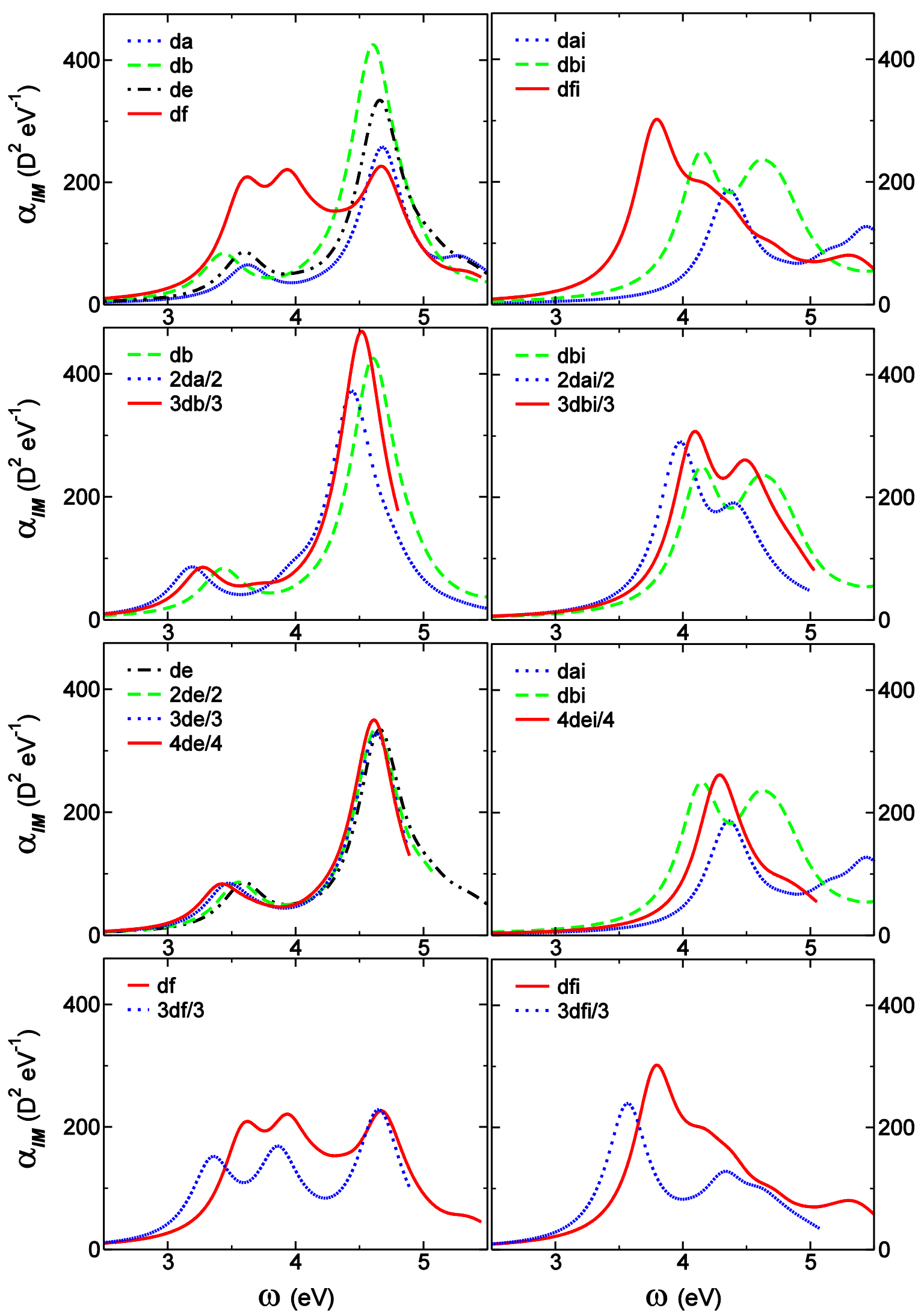

Figure 3. Calculated one photon absorption (OPA) spectra, normalized for the number of branches: nd series (Left panels), ndi series (Right panels). 


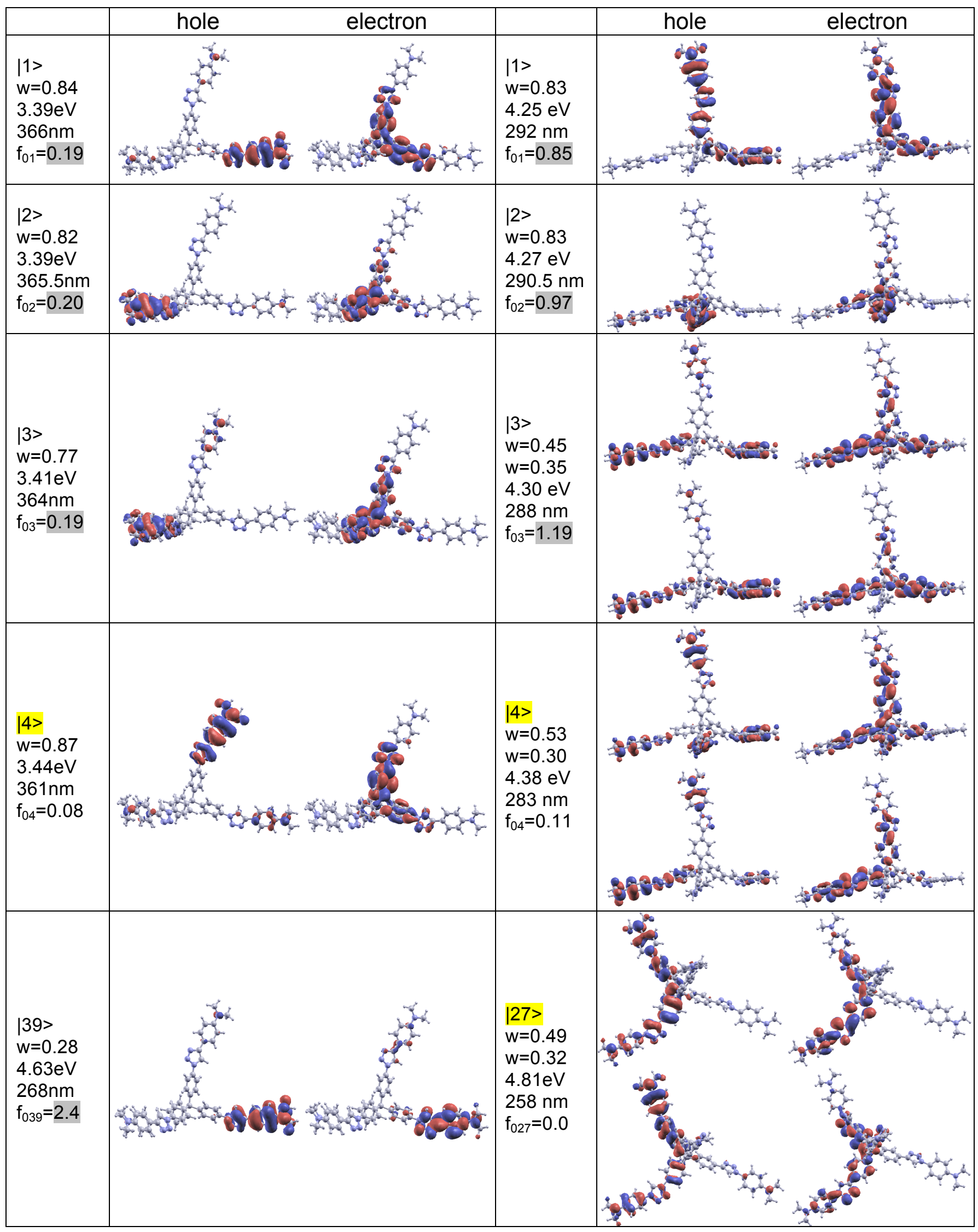

Figure 4. Natural transition orbitals ${ }^{68}$ of 3de (Left panels) and 3dei (Right panels). Text quote in sequence excited state number, associated eigenvalues, transition energies, transition wavelengths and oscillator strengths. 
J. Chem. Theory Comput., 2010, 6 (11), pp 3410-3426 ; DOI: 10.1021/ct1004406

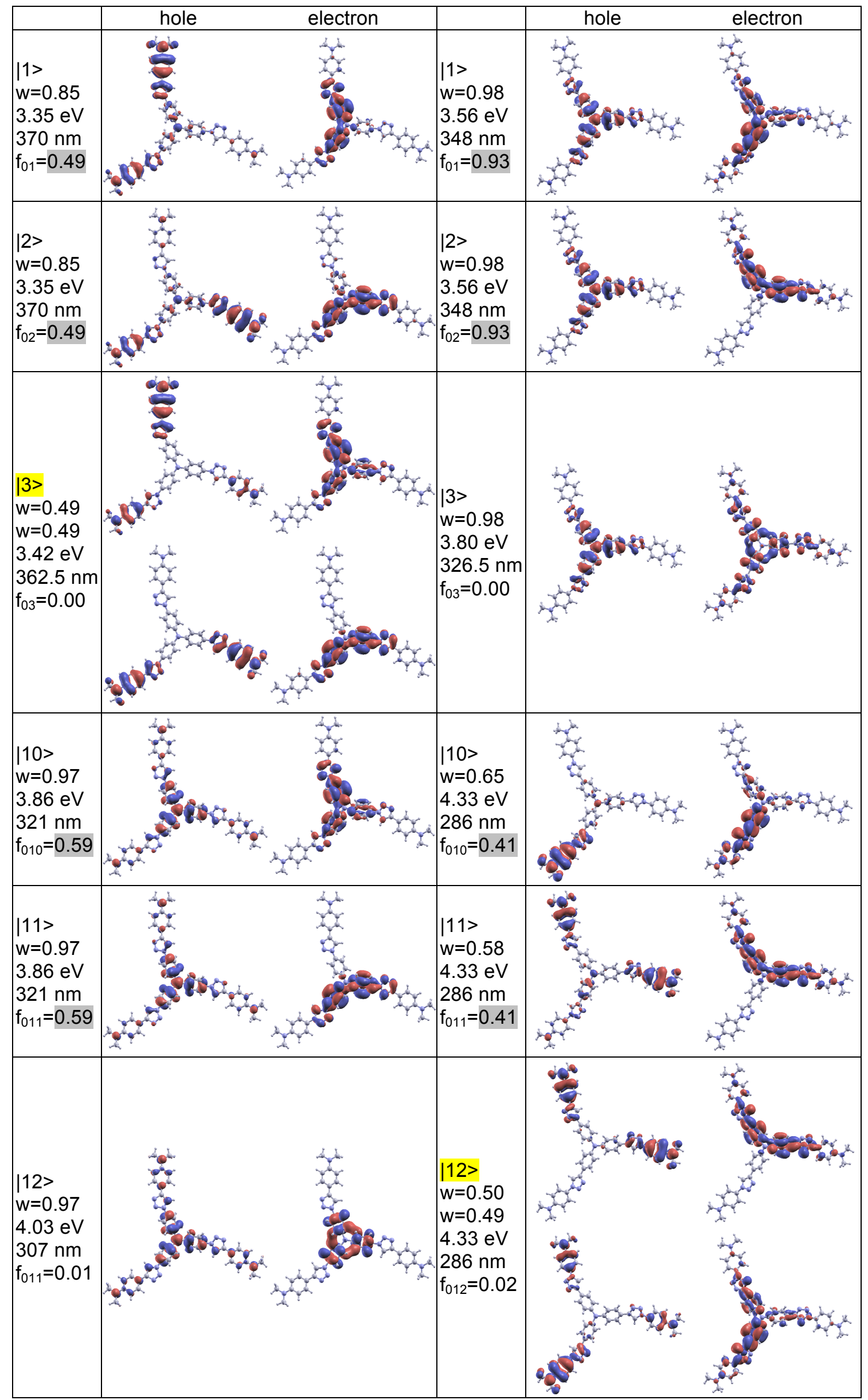


Figure 5. Natural transition orbitals ${ }^{68}$ of $3 \mathbf{d f}$ (Left panels) and 3dfi (Right panels).Text quote in sequence excited state number, associated eigenvalues, transition energies, transition wavelengths and oscillator strengths.

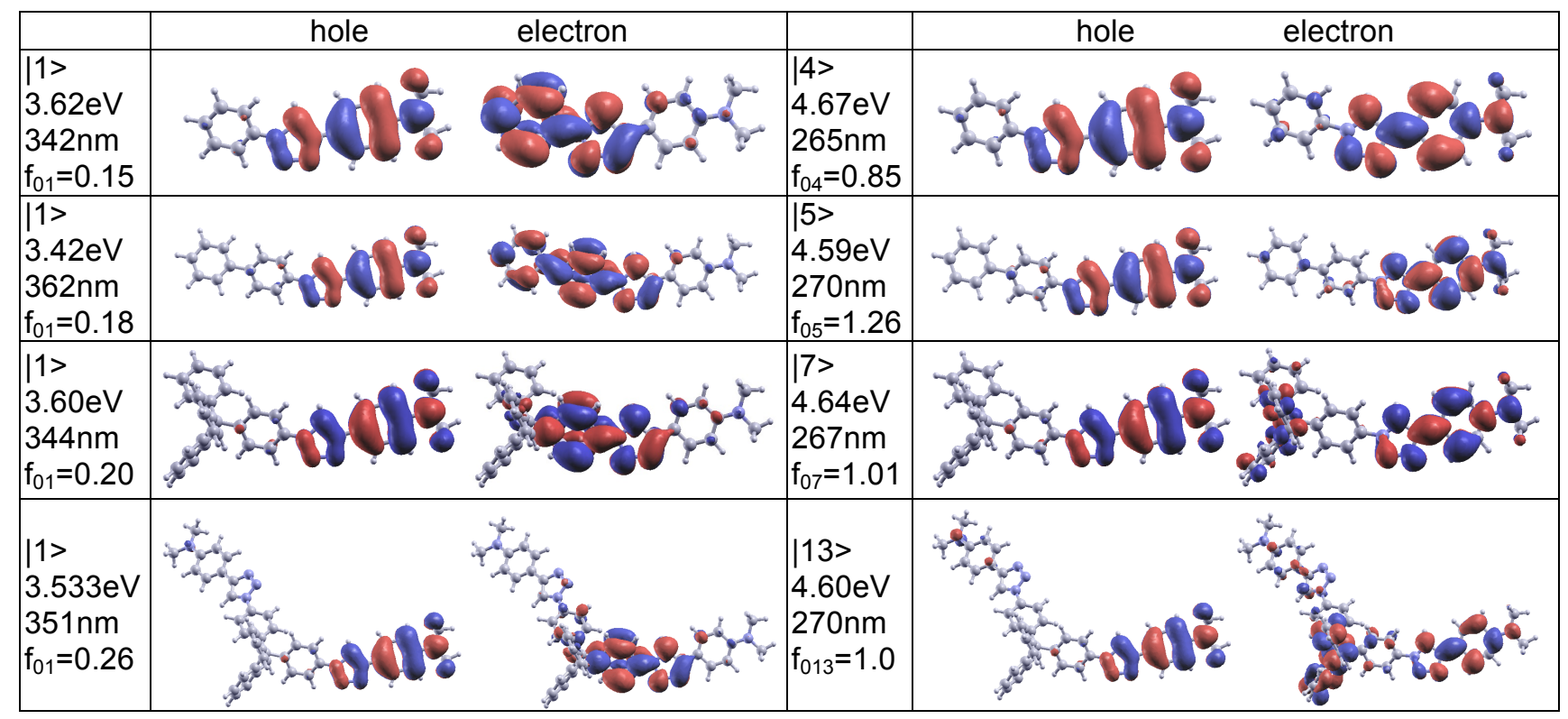

Figure 6. Natural transition orbitals ${ }^{68}$ relevant for the first (Left panels) and main (right panels) one photon absorption (OPA) band of da (top), $\mathbf{d b}$ (middle up), de (middle down) and 2de (bottom). Text quote in sequence excited state number, associated transition energies, transition wavelengths and oscillator strengths. 


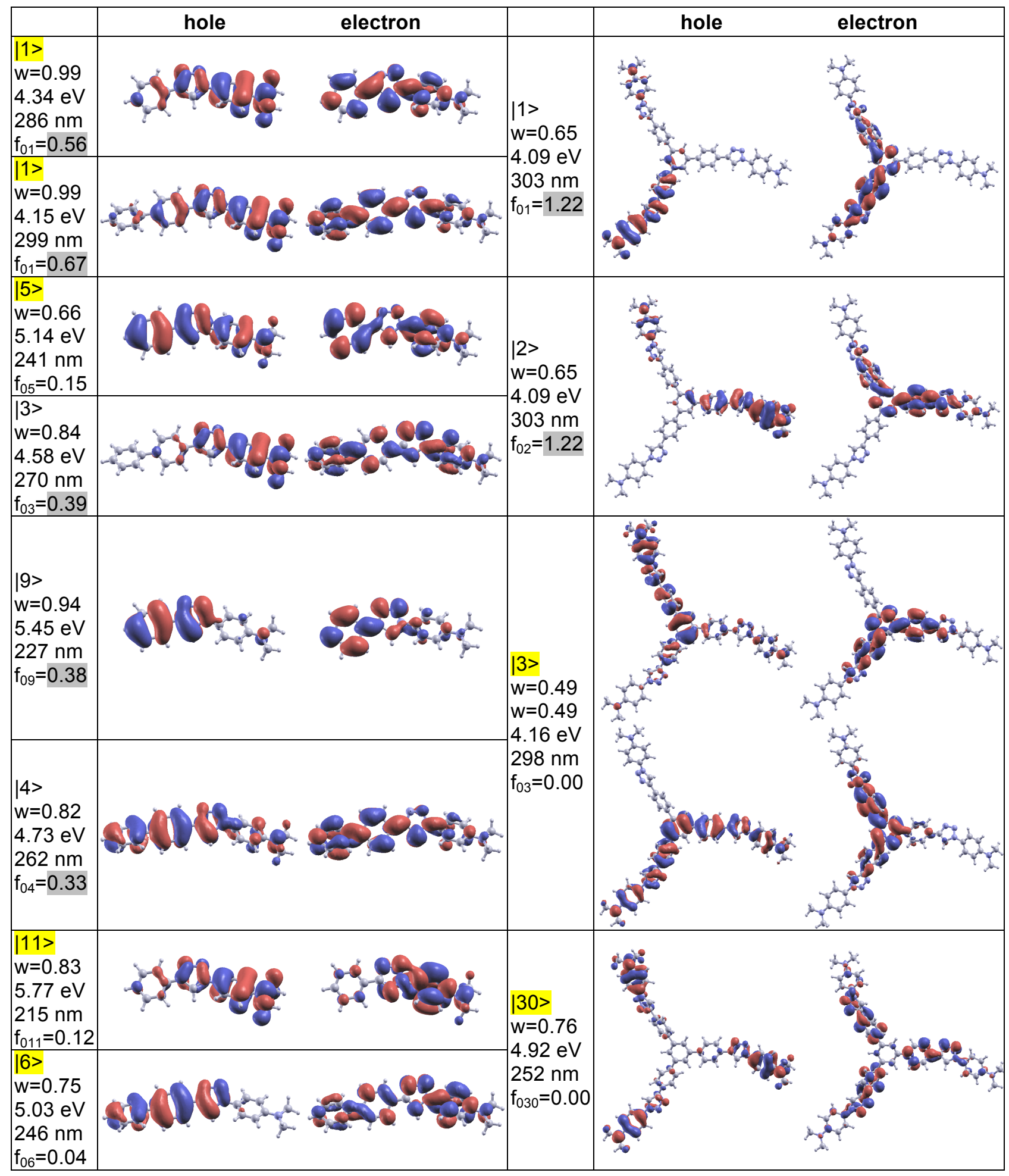

Figure 7. Natural transition orbitals ${ }^{68}$ of dai (Left panels, first line), dbi (Left panels, second line) and 3dbi (Right panels).Text quote in sequence excited state number, associated eigenvalues, transition energies, transition wavelengths and oscillator strengths. 


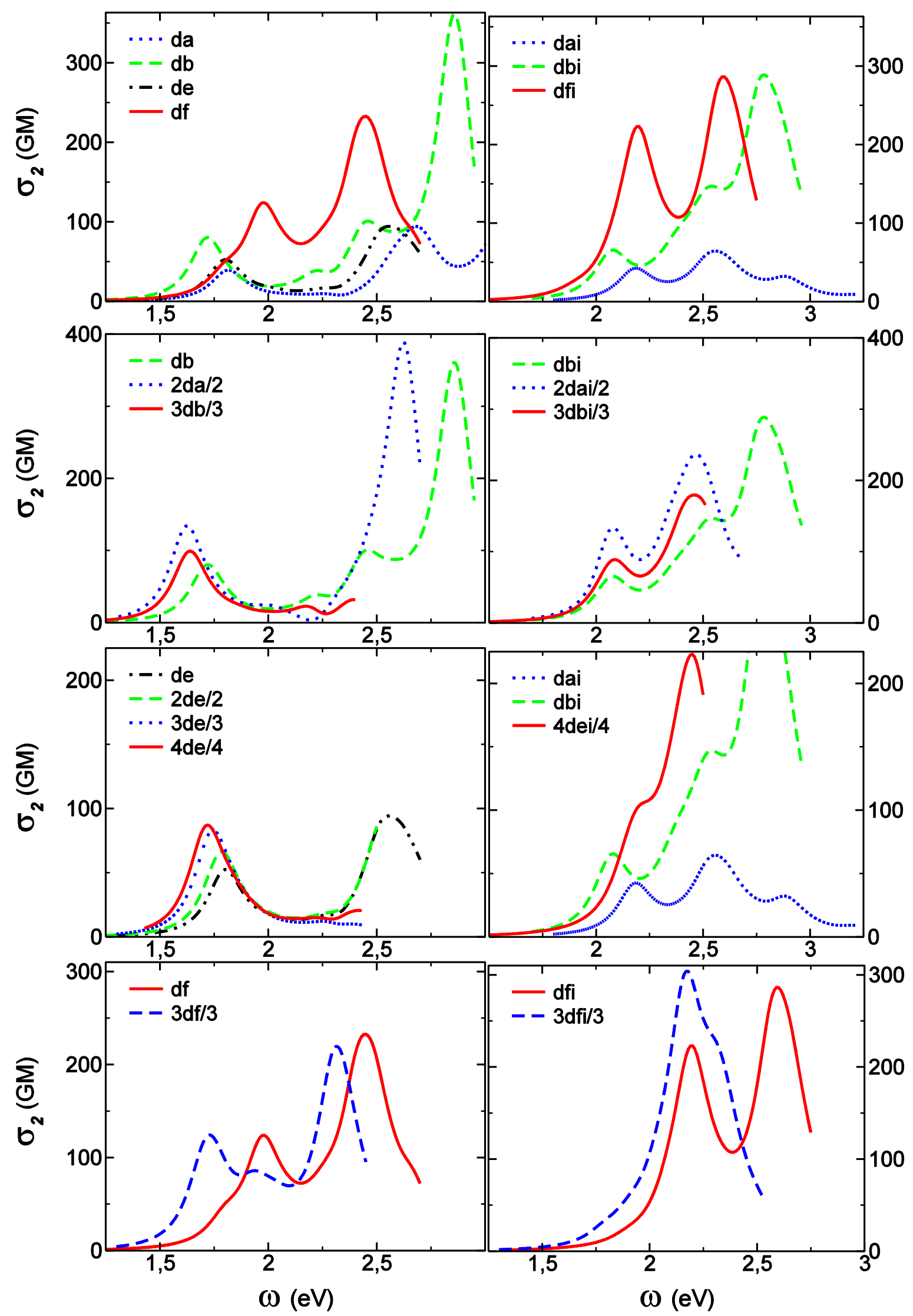

Figure 8. Calculated two photon absorption (TPA) spectra, normalized for the number of branches: nd series (Left panels), ndi series (Right panels). 